\title{
Combination with cooling-heat Chinese medicine Qing-kai-ling, not warming-yang Shen-fu, enhances the antitumor effect of gefitinib in resistant non- small cell lung cancer models in vitro and in vivo
}

\section{Yan-chun Qu}

The Second Affiliated Hospital of Guangzhou University of Chinese Medicine https://orcid.org/00000003-4959-8050

\section{Ying Zou}

Guangzhou Medical University Affiliated Cancer Hospital

\section{Shuai Shi}

Luoyang First People's Hospital

Yanjuan Zhu ( $\square$ zyjsophy@gzucm.edu.cn )

https://orcid.org/0000-0002-1956-7774

\section{Yi-hong Liu}

The Second Affiliated Hospital of Guangzhou University of Chinese Medicine

\section{Li-rong Liu}

The Second Affiliated Hospital of Guangzhou University of Chinese Medicine

\section{Xiao-hua Zheng}

Conghua Hospital of Traditional Chinese Medicine

\section{Hui-hui Chen}

Zhuhai Hospital of Integrated Traditional Chinese and Western Medicine

\section{Hai-bo Zhang}

The Second Affiliated Hospital of Guangzhou University of Chines Medicine

\section{Research}

Keywords: Traditional Chinese medicine, Treatment principle, Warming-yang treatment principle, Coolingheat treatment principle, Non-small cell lung cancer, Epidermal growth factor receptor tyrosine kinase inhibitor

Posted Date: May 21st, 2020

DOI: https://doi.org/10.21203/rs.3.rs-29063/v1 
License: (c) (i) This work is licensed under a Creative Commons Attribution 4.0 International License. Read Full License 


\section{Abstract}

Background: Traditional Chinese Medicine (TCM) prescriptions should be decided according to the TCM treatment principle, and the warming-yang or cooling-heat should be the guide of treatment principle outline.

Methods: In order to identify which treatment principle, warming-yang or cooling-heat should be combined with epidermal growth factor receptor tyrosine kinase inhibitors (EGFR-TKIs) in non-small cell lung cancer (NSCLC), we combined the typical warming-yang drug, Shen-fu (SF), and the typical coolingheat drug, Qing-kai-ling (QKL) with gefitinib in resistant NSCLC models.

Results: The results demonstrated that QKL combined with gefitinib induced significantly increased cell viability inhibition and apoptosis in A549 cell line and significantly smaller tumor volume and lower tumor weight in $\mathrm{H} 1975$ xenograft transplanted nude mice. On the contrary, SF combined with gefitinib had significant antagonism effect on both cell viability inhibition and apoptosis in vitro, and on tumor weight in vivo. EGFR phosphorylation inhibition and the downstream PI3K/AKT and RAS/RAF/ERK pathway inhibition served an important role in the synergism effect between QKL and gefitinib in H1975 xenograft transplanted nude mice.

Conclusions: The present study indicated that cooling-heat TCM treatment principle may reverse or delay NSCLC resistance to EGFR-TKIs, and combination of them warrants further study.

\section{Background}

Epidermal growth factor receptor tyrosine kinase inhibitors (EGFR-TKIs) are the preferred treatment for intermediate to advanced stage non-small cell lung cancer (NSCLC) in patients with EGFR gene mutation. Despite the demonstrated benefits of EGFR-TKIs, not all patients respond to treatment. Moreover, even for the exclusively EGFR-mutant advanced NSCLC patients, the median progression free survival (PFS) was only 9-13 months for those who accepted first-line EGFR-TKI therapy ${ }^{1}$. Approximately $50 \%$ of patients who respond well initially to TKIs develop resistance due to the occurrence of secondary mutation T790M in exon20 of the EGFR gene ${ }^{1}$, and the third generation EGFR-TKIs have shown efficacy in these patients 2,3. For the other half of the patients resistant to EGFR-TKIs, no optimal therapy was available. Even for the patients with T790M mutation, most of them are not taking third generation EGFR-TKIs in the real world Chinese clinic, because of the high price. Therefore, effective therapies to delay the resistance of EGFR-TKIs are therefore required.

TCM therapies have been widely used in cancer, including NSCLC. Although some clinical trials have reported the efficacy of some TCM decoction or patent prescription in combination with EGFR-TKIs, most of them were with small samples and low quality ${ }^{4}$. Besides, the TCM treatment principles in these studies were even different. As a result, most of the TCM therapies in clinical practice are still based on doctors' experience, rather than evidence from clinical trials. A typical TCM treatment process should be 
programmed as the following sequential steps, first diagnosing TCM syndrome types, then deciding the TCM treatment principle and finally writing out a TCM prescription. Although the syndrome types in the TCM theory are complicated, Yin-cold $(Y C)$ or Yang-heat $(Y H)$ type differentiation is believed as the outline of TCM syndrome type diagnosis according to the traditional book Huangdi Neijing. Therefore, the warming-yang or cooling-heat should be the guide of treatment principle outline ${ }^{5}$. We have demonstrated that EGFR gene mutated NSCLC patients are more likely with $Y C$ syndrome type ${ }^{6}$. Besides, the most common side effect of EGFR-TKIs was red acneiform rashes ${ }^{7-11}$, with thirsty, red and dry tongue and yellow tongue coating, which are typical symptoms and signs for $Y H$ syndrome type. Therefore, EGFRTKIs may affect with warming-yang influence according to TCM theory, and we do not know whether the warming-yang influence of EGFR-TKIs is a treatment effect or just a kind of side effect. If the warmingyang influence is a therapeutic effect, then TCM therapies with warming-yang principles may help to improve the efficacy of EGFR-TKIs, and vice versa.

In this study, we attempted to know which treatment principle, cooling-heat or warming-yang, should be combined with the EGFR-TKIs. We used the typical warming-yang drug, Shen-fu (SF), and the typical cooling-heat drug, Qing-kai-ling (QKL), because they are without known anticancer effect. SF injection has been used for nearly 30 years in China for patients with $Y C$-syndrome type. The active components of SF injection are extracted from Radix Ginseng and Radix Aconiti Carmichaeli. QKL injection or oral solution has been widely used for the treatment of high fever or acute infection in clinical practice. It is prepared from cholic acid, hyodeoxycholic acid, baicalin, and active materials extracted from Gardeniae Fructus, Bubali Cornu, Margaritifera Concha, Isatidis Radix, and Lonicerae Japonicae Flos. The aim of the present study was to investigate the effect of SF or QKL when combining with gefitinib on resistant NSCLC models in vitro and in vivo.

\section{Materials And Methods Preparation of drugs}

Gefitinib was kindly provided by AstraZeneca China. SF injection was purchased from Ya-an San-jiu pharmaceutical co., LTD (Sichuan, China), and QKL injection and QKL oral solution were purchased from Ming-xing pharmaceutical co., LTD (Guangzhou, China). The component herbs of SF decoction used in the in vivo experiments, prepared Radix Aconiti Carmichaeli and Radix Ginseng, were purchased from Kang-mei pharmaceutical co., LTD (Guangzhou, China). Seventy-five gram of Radix Ginseng and $150 \mathrm{~g}$ prepared Radix Aconiti Carmichaeli were mixed and first soaked in $1000 \mathrm{ml}$ water for 30 minutes, and then boiled for 90 minutes. The liquid was filtered through a piece of medical gauze and the drugs were boiled once more with $800 \mathrm{ml}$ water for 90 minutes. The liquid was filtered again and mixed with that from the first boiling. The solution was concentrated into $250 \mathrm{ml}$ in the rotary evaporator (IKA®RV 10 Basic), with a concentration of $0.9 \mathrm{~g} / \mathrm{ml}$ crude drug, and then stored at $-80^{\circ} \mathrm{C}$ until use.

\section{Reagents}


4,5-dimethylthiazol-2, 5-diphenyl-2-H-tetrazolium bromide (MTT) was purchased from MP Biomedicals (California, USA). Annexin V/ propidium iodide (PI) apoptosis Kit was purchased from MultiSciences(Lianke)Biotech Co., Ltd (Hangzhou, China). BCA protein assay kit was purchased from Thermo Fisher Scientific, Inc ( ML, USA). Rabbit anti-human EGFR, phospho-EGFR (p-EGFR), AKT, p-AKT, ERK and $p$-ERK monoclonal antibodies $(\mathrm{mAb})$ and horseradish peroxidase $\square \mathrm{HRP} \otimes$ conjugated anti-rabbit antibody were purchased from Cell Signaling Technology, Inc (MA, USA). Electro-Chemi-Luminescence (ECL) reagent was purchased from Millipore Corporation (MA, USA).

\section{Cell Culture}

Human A549 NSCLC cells were obtained from the Cell Line Bank at the Laboratory Animal Center of Sun Yat-sen University (Guangzhou, China). H1975 cells were obtained from the Cell Line Bank of the Macao University of Science and Technology (Macao, China). Cells were maintained in RPMI-1640 medium (Gibco; ThermoFisher Scientific, Inc., MA, USA) supplemented with 10\% FBS (Gibco; Thermo Fisher Scientific, Inc.) and $0.5 \%$ penicillin-streptomycin sulfate (Gibco; Thermo Fisher Scientific, Inc.), and incubated at $37 \mathrm{C}$ with $5 \% \mathrm{CO}^{2}$.

\section{Cell Viability Assay}

Human A549 cell was used in the in vitro experiments. MTT assay was used to measure cell viability. Briefly, cells were plated in 96-well culture plates at the density of $5 \times 10^{3}$ per well in complete medium. After 24 hours of incubation, cells were treated with gefitinib (20-100 $\mu \mathrm{M})$, QKL injection and SF injection (0.6\%-1.0\%) alone or combination for 48 and 72 hours. Then the cells were incubated with $110 \mu$ of $5 \mathrm{mg} / \mathrm{ml} \mathrm{MTT}$ at $37^{\circ} \mathrm{C}$ for $4 \mathrm{~h}$. After removing the medium, $150 \mu \mathrm{l}$ dimethylsulfoxide (DMSO, Sigma, MO, USA) was added to each well. After shaking for $10 \mathrm{~min}$, the absorbance at $570 \mathrm{~nm}$ was measured spectrophotometrically using a microplate reader (VICTOR X5,PerkinElmer). Each condition was duplicated in 6 wells. After removing the maximum and minimum absorbance to calculate the average, the cellular viability in each condition was expressed as the percentage of the average absorbance to that of control cells.

\section{Apoptosis Assay}

Apoptotic cell death was determined by the Annexin V/PI apoptosis Kit according to the manufacturer's instructions. Briefly, cells were plated in 6-well culture plates at a density of $1.5 \times 10^{6} \mathrm{cells} / \mathrm{mL}$ in complete medium. After 24 hours of incubation, cells were treated with gefitinib, QKL and SF injection alone or combination, with the concentration and exposure time according to the MTT assay results. After removing the medium, cells were trypsinized with EDTA free trypsin solution (Gibco; Thermo Fisher Scientific, Inc.), harvested and then resuspended in $500 \mu$ l Binding Buffer (1x) with $5 \mu$ I Annexin V-FITC 
and $10 \mu \mathrm{PI}$. After incubation for $5 \mathrm{~min}$ at room temperature in the dark, the samples were analyzed with flow cytometer (Beckman Coulter FC500).

\section{Western Blot Analysis}

The cells were plated in $60 \mathrm{~mm}$ dishes at a density of $3.5 \times 10^{6} \mathrm{cells} / \mathrm{ml}$ in complete medium. After 24 hours of incubation, cells were treated with gefitinib, QKL and SF injection alone or combination, with the concentration and exposure time according to the MTT assay results. After the treatment, the cells were washed in PBS and lysed in lysis buffer. Protein concentrations were determined using the BCA protein assay kit. The samples corresponding to $30 \mu \mathrm{g}$ of protein were boiled for $8-10 \mathrm{~min}$, resolved on an $8 \%$ denatured SDS-polyacrylamide gel, and transferred onto a PVDF membrane (Millipore, MA, USA). After blocking non-specific binding sites for 30 minutes using 5\% skim milk, the membranes were incubated with rabbit anti-human EGFR, p-EGFR, AKT, p-AKT, ERK and p-ERK monoclonal antibodies for 2 hours at room temperature. After washed with TBST for 3 times, the membranes were incubated with HRP conjugated anti-rabbit antibody for 1 hour at room temperature. After washed with TBST for 3 times, visualization of the protein bands was accomplished using ECL reagent. ImageLab software (version 4.0) was used to calculate the expression of each protein, which was normalized by GAPDH.

\section{Determination of antitumor effect in nude mice.}

Female BALB/c nude mice (18-20 g) were obtained from the Laboratory Animal Center of Southern Medical University (Guangzhou, China, License NO. 44002100006205). The animals were kept in the Animal Center of Guangdong Provincial Hospital of Chinese Medicine (License NO. SYXK(yue)20130094), under a specific pathogen-free (SPF) condition with a $12 \mathrm{~h}$ light/dark cycle and freely accessed autoclaved standard food and water. Human NSCLC cells H1975 $\left(3 \times 10^{7} / \mathrm{ml}\right)$ were suspended in RPMI1640 medium and $0.2 \mathrm{ml}$ of the suspension was subcutaneously inoculated into the right forelimb of nude mice. Tumor growth was measured with the longest diameter (a) and the shortest diameter vertical to a (b). Tumor volume was calculated using the formula, $v=\prod a b^{2} / 6$.

When the tumors reached the size over $150 \mathrm{~mm}^{3}$, the mice were randomly divided into 6 groups $(n=10)$ : control (saline solution $0.2 \mathrm{ml}$ ), gefitinib (1 mg, in $0.2 \mathrm{ml}$ saline solution), QKL oral solution ( $0.25 \mathrm{ml})$, SF decoction (0.2 ml), gefitinib + SF, and gefitinib + QKL. The compounds were administered orally once a day for consecutive 21 days. Tumor volume and body weight of the mice were measured every 3 days during the administration period. The daily dosage of each drug for nude mice (with average weight of $20 \mathrm{~g}$ ) was obtained based on the daily dosage for humans in clinical and the human-mouse transfer formula: Animal dose = Human dose $\mathrm{x}(\mathrm{HKm} / \mathrm{AKm})$, where $\mathrm{HKm}$ and AKm represent the $\mathrm{Km}$ factor of human (37) and mouse (3) ${ }^{12}$. The daily dosages for humans (with average weight of $60 \mathrm{~kg}$ ) are $250 \mathrm{mg}$ of gefitinib, $60 \mathrm{ml}$ of QKL oral solution, and $45 \mathrm{~g}$ crude drug of SF decoction.

\section{Immunohistochemistry}


For immunohistochemical staining, paraffin-embedded tumor tissues were applied. The sections were deparaffinized in xylene and rehydrated with graded alcohol. Hydrogen peroxide (3\%) was applied to block endogenous peroxide activity and then boiled in $0.01 \mathrm{M}$ citrate buffer $(\mathrm{pH} 6.0)$ twice with an autoclave. After blocking non-specific binding sites using normal goat serum (Boster Biological Technology co.Itd, California, USA), tissue sections were incubated with rabbit anti-human EGFR (1:100), p-EGFR (1:100), AKT (1:200), p-AKT (1:50), ERK (1:200) or p-ERK (1:100) monoclonal antibodies at $4^{\circ} \mathrm{C}$ overnight. After washed with phosphate buffer saline (PBS) for 3 times, the sections were incubated with HRP conjugated anti-rabbit antibody for 30 minutes at $37^{\circ} \mathrm{C}$, and the peroxidase reaction was developed with diaminobenzidine substrate kit (Zhongshan Golden Bridge-Bio, Beijing, China). Hematoxylin (Dingguo Changsheng Biotechnology CO., Ltd, Beijing, China) was then used for nucleus staining. ImageProPlus 5.0 software was used to calculate the ratio of integrated optical density (IOD) to area (IOD/Area).

\section{Statistics}

Statistical analysis was performed using SPSS 19.0 statistical software (SPSS, Inc., Chicago, USA). The in vitro experiments were performed three times, independently. All data were presented as the mean \pm standard deviation (SD). Differences between groups were assessed by two-tailed $t$ test, one-way analysis of variance or analysis of variance for repeated measuring data and least significant difference (LSD)-t test was used for multiple comparisons. $\mathrm{P}<0.05$ was considered to indicate a statistically significant difference. $q$ value method was used to evaluate the combination effect of gefitinib and QKL/SF, and it was calculated using the equation: $q=E A B /(E A+E B-E A \times E B)$, where $E A$ and $E B$ were the inhibition effect of gefitinib and QKL/SF, respectively. $E A B$ represented an observed value of combined effect, and (EA + EB- EA $\times$ EB) represented an expected value of combine effect. A $q$ value of 1.15 or more is considered synergism, $q<0.85$ as antagonism and the value between 0.85 and 1.1 .5 is considered as additive effect ${ }^{13}$.

\section{Results}

\section{Cell viability in vitro}

We first screened the concentration of gefitinib. According to the MTT assay, $45 \mu \mathrm{M}$ of gefitinib for A549 was used in the experiments with 48 hours of drug exposure, and $35 \mu \mathrm{M}$ for A549 was used in the cells exposed to the drugs for 72 hours. As shown in Fig. 1, in A549 cell lines, the $0.9 \%$ and $1.0 \%$ of QKL injection combined with gefitinib induced significantly increased viability inhibition after 72 hours treatment compared with gefitinib alone. However, the q value indicated only additive not synergism effect $(0.85<q<1.15)$. There were no significant viability differences between gefitinib alone and SF + gefitinib treated $A 549$ cells. According to the maximum combination effect and minimum effect of QKL or SF injection alone in MTT assay, $35 \mu \mathrm{M}$ of gefitinib and $0.9 \%$ of SF or QKL injection with 72 hours in A549 was used in the apoptosis and western blot experiments. 
Synergism effect of QKL injection and gefitinib on apoptosis were seen in the A549 ( $q=1.40)$ cell line, with significantly increased apoptosis rate in the QKL + gefitinib treated cells compared with the gefitinib treated cells (Fig. 2). On the contrary, although the differences in apoptosis rates were not significant between the SF + gefitinib and gefitinib alone treated cells, q value method showed antagonism effect of $S F$ injection and gefitinib in A549 ( $q=0.75)$.

\section{Antitumor tumor activity in vivo}

In H1975 xenograft transplanted nude mice, QKL oral solution, gefitinib, and QKL + gefitinib inhibited tumor growth, with significantly smaller tumor volume and lower tumor weight compared with those in control group $(p<0.05$, Fig. 3$)$. Differences in tumor volume and weight among SF decoction, SF + gefitinib and control group were not significant. Tumor volume and weight in QKL + gefitinib group were even significantly lower than in gefitinib group $(p<0.05)$. $Q$ value according to the inhibition rate in tumor weight indicated synergism effect between QKL and gefitinib ( $q=1.19)$. Although the differences in tumor volume and weight between SF + gefitinib and gefitinib groups were not significant, q value shown antagonism effect between SF and gefitinib $(q=0.50)$.

\section{EGFR Pathway Protein Expression}

In the experiments in vitro, the protein expression levels of p-EGFR were significantly lower in gefitinib, gefitinib + SF, and gefitinib + QKL groups in A549 cell line, comparing with control groups (Fig. 4A). The differences among the three groups were not significant. No significant differences were seen in EGFR, AKT/p-AKT, or ERK/p-ERK protein expression among A549 groups.

In H1975 xenograft transplanted nude mice, p-EGFR, AKT/p-AKT and p-ERK protein levels were significantly lower in gefitinib + QKL treated group, comparing with control group (Fig. 4B). Differences in EGFR and AKT/p-AKT protein levels were also significant between gefitinib alone and gefitinib + QKL treated groups. These data suggested that EGFR phosphorylation inhibition and the downstream $\mathrm{PI} 3 \mathrm{~K} / \mathrm{AKT}$ and RAS/RAF/ERK pathway inhibition served an important role in the synergism effect between QKL oral solution and gefitinib in $\mathrm{H} 1975$ xenograft transplanted nude mice. Although EGFR protein level in gefitinib + SF group was significantly increased, comparing with control group, there were no significant differences in p-EGFR or downstream protein levels. Therefore, mechanism of antagonism effect between SF decoction and gefitinib was not clear.

\section{Discussion}

EGFR-TKIs are the preferred treatment for intermediate to advanced stage NSCLC in patients with EGFR gene mutation. However, the median PFS was only 9-13 months even for the exclusively EGFR-mutant advanced NSCLC patients. More effective therapies are therefore required. Traditional Chinese medicine, 
which is popular in Chinese and East Asian societies, may be a potential effective strategy to delay the resistance to EGFR-TKIs.

The present study demonstrated that in gefitinib resistant in vitro and in vivo models, cooling-heat TCM prescription and gefitinib had synergism effect, while warming-yang prescription was antagonistic to gefitinib. Some clinical studies also indicated that TCM prescriptions or decoction with cooling-heat treatment principle may increase the efficacy of first-generation EGFR-TKIs. In Yang X's study, the TCM treatment principle of the decoction was strengthening vital qi and cooling cancer toxicity, especially cooling heat, and TCM decoction combining with gefitinib significantly prolonged progression free survival and overall survival ${ }^{14}$. In He W's meta-analysis, which analyzed the Chinese studies published from 2000 to 2016, only 6 studies reported increased efficacy from TCM when combining with EGFRTKIs. Four of these 6 studies used specific TCM patent prescription or decoction, in which 3 were with cooling-heat treatment principle ${ }^{4}$. On the other hand, a case report by Sung-Wook Hwang et al. reported that inappropriate TCM herbs, mainly Ginseng with warming-yang effect, induced resistance to gefitinib whereas withdrawing of the herbs caused sensitivity again to gefitinib ${ }^{15}$.All these studies, together with our findings, suggested that the observed warming-yang effect of EGFR-TKls may be a kind of side-effect even in TCM theory, and should be combined with cooling-heat treatment principle TCM therapies.

We also tried to find out the mechanism of the synergism or antagonism effect of QKL or SF to gefitinib. However, we only demonstrated that EGFR phosphorylation inhibition served an important role in the synergism effect between QKL oral solution and gefitinib in H1975 xenograft nude mice. Interestingly, $\mathrm{H} 1975$ cells are refractory to EGFR-TKIs due to the presence of the T790M mutation in EGFR ${ }^{16}$, which is the mechanism of $50 \%$ of the EGFR-TKIs resistant patients ${ }^{1}$. The T790M mutation results in steric hindrance of binding of gefitinib to the ATP-kinase-binding pocket ${ }^{16}$. Our data suggested that QKL induced the EGFR phosphorylation re-inhibition and downstream PI3K/AKT and RAS/RAF/ERK pathway re-inhibition of gefitinib. This re-inhibition in T790M mutant $\mathrm{H} 1975$ may resulted from steric re-binding of gefitinib to the ATP-kinase-binding pocket, because downstream pathway re-inhibition was not observed in A549 (with K-ras not EGFR mutation ${ }^{17}$ ). Therefore, protein domain structure analyses warrant further researches.

The limitation of this present study was that we used only one drug to present each of the treatment principle. Expansion of the observed synergism effect of QKL and antagonism effect of SF with gefitinib to the effect of cooling-heat or warming-yang treatment principle should be very careful. Based on our initial finding, there are some TCM issues what warrant further research. First, we can further use other TCM patent prescriptions or decoction to present the cooling-heat or warming-yang treatment principle. If similar phenomena would be observed again, our hypothesis would be confirmed. Secondly, since synergism effect of QKL with gefitinib in tumor growth and apoptosis has been demonstrated in vitro and in vivo, reversing the resistance to gefitinib, effect on epithelial-mesenchymal transition (EMT) and metastasis warrants further researches, which should also been transferred to clinical validation. All 
these researches will be meaningful for guiding the principle of TCM therapies in combination with EGFRTKIs, or for finding new ways to delay the resistance to EGFR-TKIs.

\section{Conclusions}

In conclusion, we found that QKL, with cooling-heat TCM treatment principle, increased efficacy of gefitinib in refractory models, while warming-yang SF acted as antagonist to gefitinib. The influence of other drugs with cooling-heat or warming-yang TCM treatment principle warrant further study.

\section{Abbreviations}

TCM: Traditional Chinese Medicine; EGFR-TKIs: epidermal growth factor receptor tyrosine kinase inhibitors; NSCLC: non-small cell lung cancer; SF: Shen-fu; QKL: Qing-kai-ling; PFS: progression free survival; YC: Yin-cold; YH: Yang-heat; PI: propidium iodide; p-EGFR: phospho-EGFR; mAb: monoclonal antibodies; HRP: horseradish peroxidase; ECL: Electro-Chemi-Luminescence; PBS: phosphate buffer saline; SPF: specific pathogen-free; HKm: the Km factor of human; AKm: the Km factor of animal; IOD: integrated optical density; SD: standard deviation; LSD: least significant difference; EMT: epithelialmesenchymal transition.

\section{Declarations}

\section{Acknowledgements}

Not applicable.

\section{Authors'contributions}

Yan-juan Zhu and Hai-bo Zhang designed the study; Yan-chun Qu and Yan-juan Zhu wrote the main manuscript text; Ying Zou, Shuai Shi, Xiao-hua Zheng, Hui-hui Chen performed the cell and animal experiments; Yan-chun Qu, Yi-hong Liu, Li-rong Liu analysed the data and prepared the figures. All authors read and approved the final manuscript.

\section{Funding Sources}

This work was supported by the Foundation for Chinese Medicine and Technology Researches of Guangdong Provincial Hospital of Traditional Chinese Medicine (YK2013B2N07, 2017KT1332), and Wu jieping medical foundation (320.6750.15085).

\section{Availability of data and materials}

The datasets used and/or analyzed during the current study are available from the corresponding author on reasonable request. 


\section{Ethics approval and consent to participate}

The animal experiment protocol was approved by Animals Research Committee of Guangdong Provincial Hospital of Chinese Medicine (No.2014027).

\section{Consent for publication}

The manuscript is approved by all authors for publication.

\section{Declaration of conflicting interests}

All the authors listed have approved this manuscript. The authors declare that we have no conflict of interest.

\section{Author details}

${ }^{1}$ Oncology Department, Guangdong Provincial Hospital of Traditional Chinese Medicine, No. 111 Dade Road, Yuexiu District, Guangzhou 510120, China. ${ }^{2}$ Oncology Department, The Second Affiliated Hospital of Guangzhou University of Chinese Medicine, No.111 Dade Road, Yuexiu District, Guangzhou 510120, China. ${ }^{3}$ The Second Clinical Medical College of Guangzhou University of Chinese Medicine, No. 12 Jichang Road, Baiyun District, Guangzhou 510405, China. ${ }^{4}$ Guangdong Provincial Key Laboratory of Clinical Research on Traditional Chinese Medicine Syndrome, No. 111 Dade Road, Yuexiu District, Guangzhou 510120, China. ${ }^{5}$ Department of Integrated Traditional and Western Medicine, Affiliated Cancer Hospital \& Institute of Guangzhou Medical University, No.78 Hengzhigang Road, Yuexiu District, Guangzhou 510635, China. ${ }^{6}$ Department of Respiration Medicine, Luoyang First People's Hospital, No.88 Zhongzhou East Road, Luoyang 471000, China. ${ }^{7}$ Department of Respiration Medicine区 Conghua Hospital of Traditional Chinese Medicine, No.21, North road, Jiekou street, Conghua district, Guangzhou 510900, China. ${ }^{8}$ Zhuhai Hospital of Integrated Traditional Chinese and Western Medicine, No.208 Yuehua Road, Gongbei, Zhuhai 519000,China.

\section{References}

1. Zhong WZ, Zhou Q, Wu YL. The resistance mechanisms and treatment strategies for EGFR-mutant advanced non-small-cell lung cancer. Oncotarget. 2017;8:71358-70.

2. Cross DA, Ashton SE, Ghiorghiu S, et al. AZD9291, an irreversible EGFR TKI, overcomes T790Mmediated resistance to EGFR inhibitors in lung cancer. Cancer Discov. 2014;4:1046-61.

3. Sequist LV, Soria JC, Goldman JW, et al. Rociletinib in EGFR-mutated non-small-cell lung cancer. N Engl J Med. 2015;372:1700-9.

4. He Wei, Miao C. [Meta- analysis on effectiveness and safety of traditional Chinese medicine combined with first- generation EGFR-TKI in treating advanced non-small cell lung cancer]. China Journal of Chinese Materia Medica. 2017;42:2591-8. 
5. Li S, Lu A, Jia H. Therapeutic actions of the Chinese herbal formulae with cold and heat properties and their effects on ultrastructures of synoviocytes in rats of the collagen-induced arthritis. $J$ Tradit Chin Med. 2002;22:296-302.

6. Zhu YJ, Zhang HB, Liu LR, et al: Yin-Cold or Yang-Heat Syndrome Type of Traditional Chinese Medicine Was Associated with the Epidermal Growth Factor Receptor Gene Status in Non-Small Cell Lung Cancer Patients: Confirmation of a TCM Concept. Evidence-Based Complementary and Alternative Medicine.2017.

7. Mitsudomi T, Morita S, Yatabe Y, et al. Gefitinib versus cisplatin plus docetaxel in patients with nonsmall-cell lung cancer harbouring mutations of the epidermal growth factor receptor (WJTOG3405): an open label, randomised phase 3 trial. Lancet Oncol. 2010;11:121-8.

8. Zhou C, Wu YL, Chen G, et al. Erlotinib versus chemotherapy as first-line treatment for patients with advanced EGFR mutation-positive non-small-cell lung cancer (OPTIMAL, CTONG-0802): a multicentre, open-label, randomised, phase 3 study. Lancet Oncol. 2011;12:735-42.

9. Maemondo M, Inoue A, Kobayashi K, et al. Gefitinib or chemotherapy for non-small-cell lung cancer with mutated EGFR. N Engl J Med. 2010;362:2380-8.

10. Rosell R, Carcereny E, Gervais R, et al. Erlotinib versus standard chemotherapy as first-line treatment for European patients with advanced EGFR mutation-positive non-small-cell lung cancer (EURTAC): a multicentre, open-label, randomised phase 3 trial. Lancet Oncol. 2012;13:239-46.

11. Sequist LV, Yang JC, Yamamoto N, et al. Phase III study of afatinib or cisplatin plus pemetrexed in patients with metastatic lung adenocarcinoma with EGFR mutations. J Clin Oncol. 2013;31:332734.

12. Reagan-Shaw S, Nihal M, Ahmad N. Dose translation from animal to human studies revisited. Faseb Journal. 2008;22:659-61.

13. Liu Z, Li HF, Fan YY, et al. Combination treatment with Rhizoma Paridis and Rhizoma Curcuma longa extracts and 10-hydroxycamptothecin enhances the antitumor effect in $\mathrm{H} 22$ tumor model by increasing the plasma concentration. Biomed Pharmacother. 2016;83:627-34.

14. Yang XB, Wu WY, Long SQ, et al. Effect of gefitinib plus Chinese herbal medicine (CHM) in patients with advanced non-small-cell lung cancer: A retrospective case control study. Complementary Therapies in Medicine. 2014;22:1010-8.

15. Hwang SW, Han HS, Lim KY, et al. Drug interaction between complementary herbal medicines and gefitinib. J Thorac Oncol. 2008;3:942-3.

16. Pao W, Miller VA, Politi KA, et al. Acquired resistance of lung adenocarcinomas to gefitinib or erlotinib is associated with a second mutation in the EGFR kinase domain. Plos Medicine. 2005;2:225-35.

17. Pao W, Wang TY, Riely GJ, et al. KRAS mutations and primary resistance of lung adenocarcinomas to gefitinib or erlotinib. Plos Medicine. 2005;2:57-61.

\section{Figures}



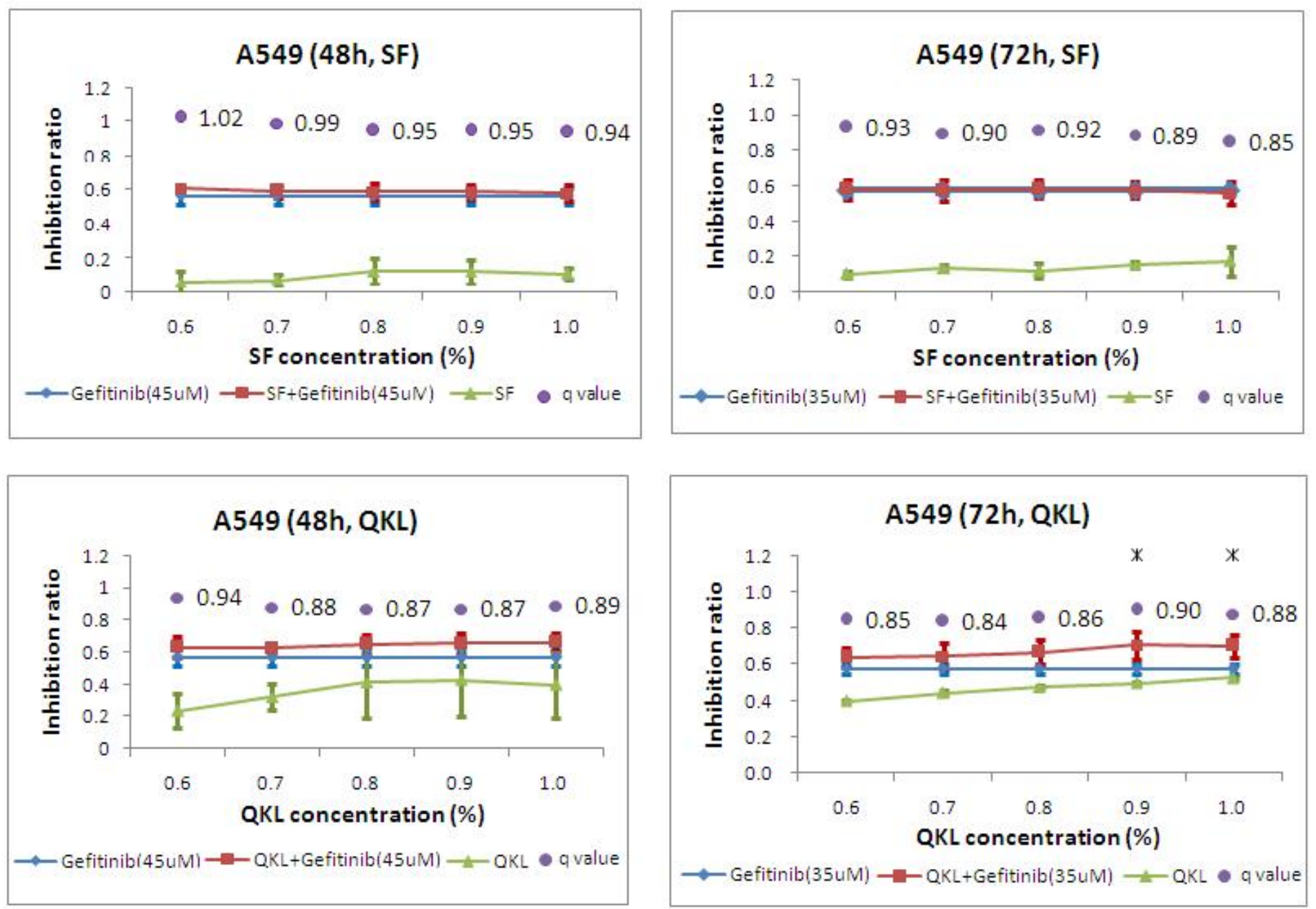

\section{Figure 1}

MTT assay indicated that Qingkailing enhanced the cell viability inhibition of gefitinib in A549. Data are presented as the mean \pm standard deviation of three independent experiments. ${ }^{*} \mathrm{P}<0.05$ vs. gefitinib alone. SF, Shenfu injection; QKL, Qingkailing injection.

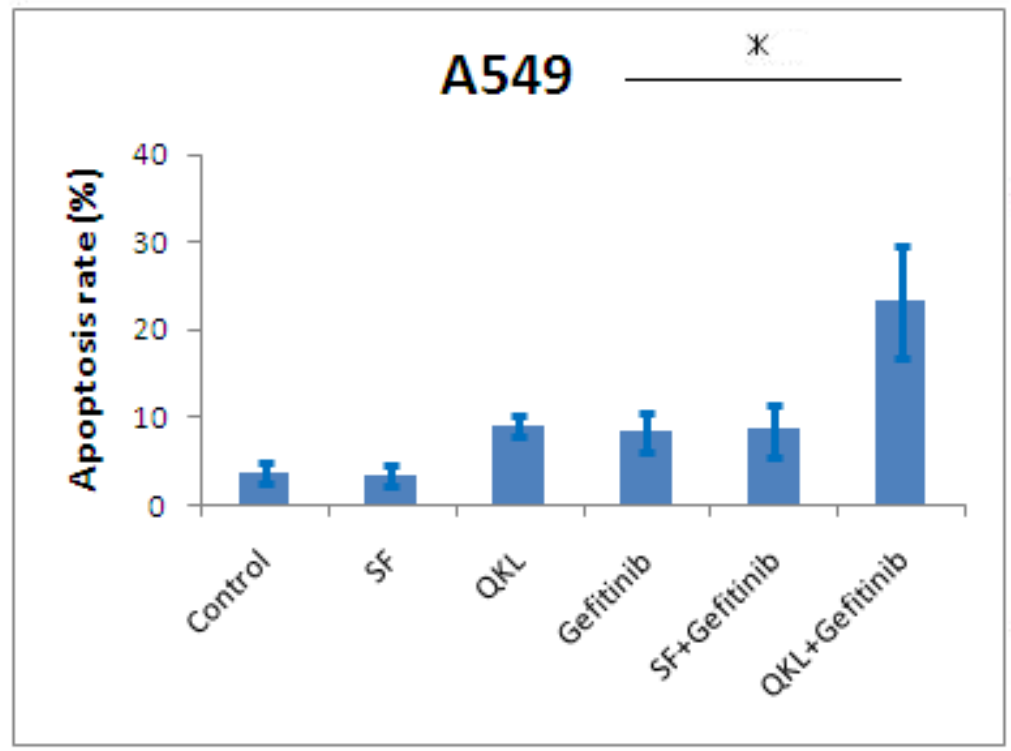


Figure 2

Apoptosis assay indicated that Qingkailing combining with gefitinib increased the apoptosis rate in A549, with synergism effect $(q=1.40)$. Shenfu and gefitinib had antagonism effect in A549 ( $q=0.75)$. Data are presented as the mean \pm standard deviation of three independent experiments. ${ }^{*} P<0.05$ vs. gefitinib alone. SF, Shenfu injection; QKL, Qingkailing injection.
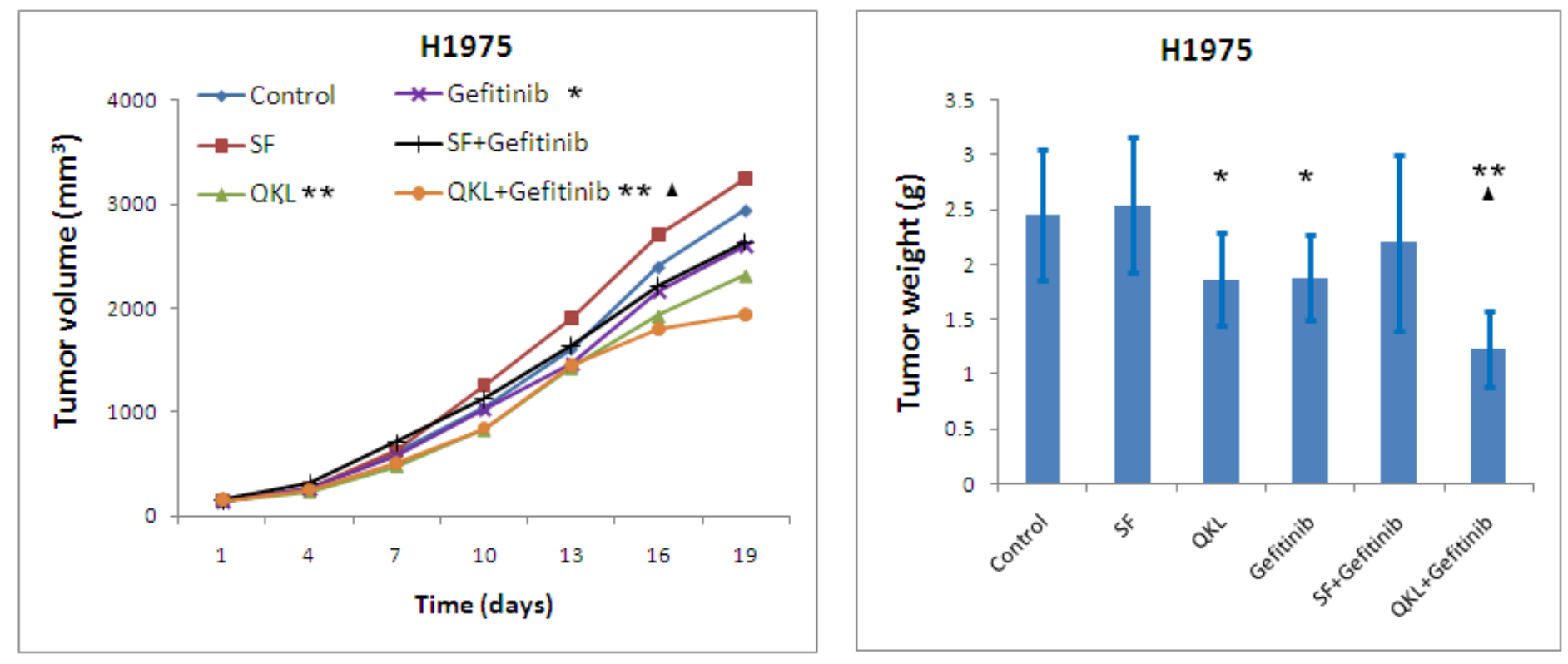

\section{Figure 3}

Qingkailing (QKL) oral solution, gefitinib, and QKL+gefitinib inhibited H1975 tumor growth in nude mice, with significantly smaller tumor volume and lower tumor weight compared with those in control group. Combining with QKL enhanced the efficacy of gefitinib, with synergism effect ( $q=1.19)$, while Shenfu decoction shown antagonism effect to gefitinib $(q=0.50)$. Data are presented as the mean \pm standard deviation. ${ }^{*} P<0.05$ and ${ }^{*} \mathrm{P}<0.01$ vs. control. $\Delta \mathrm{P}<0.05$ vs. gefitinib alone. $S F$, Shenfu decoction; $Q K L$, Qingkailing oral solution. 

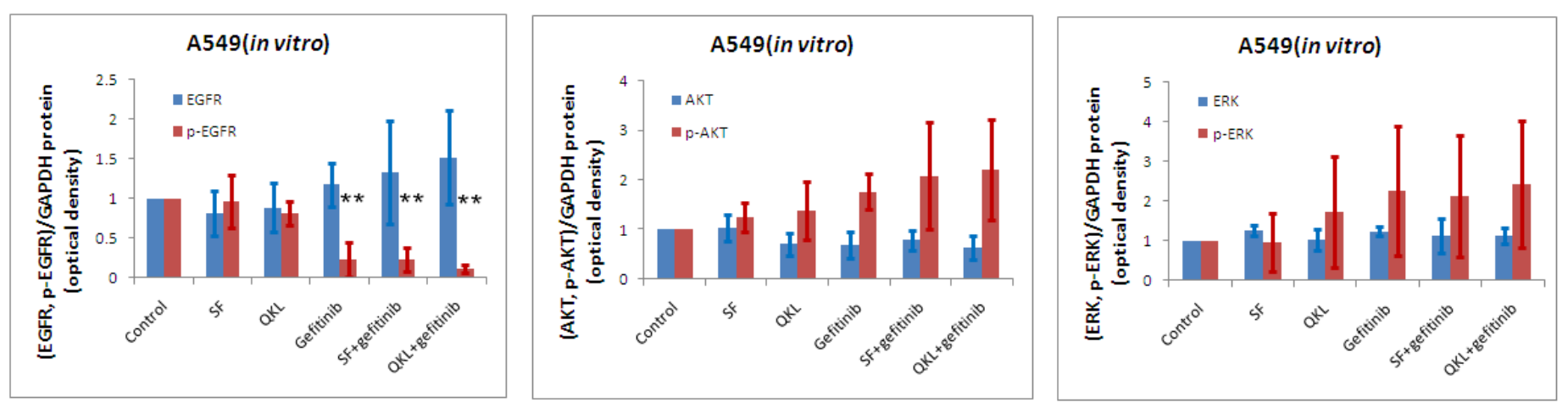

4A
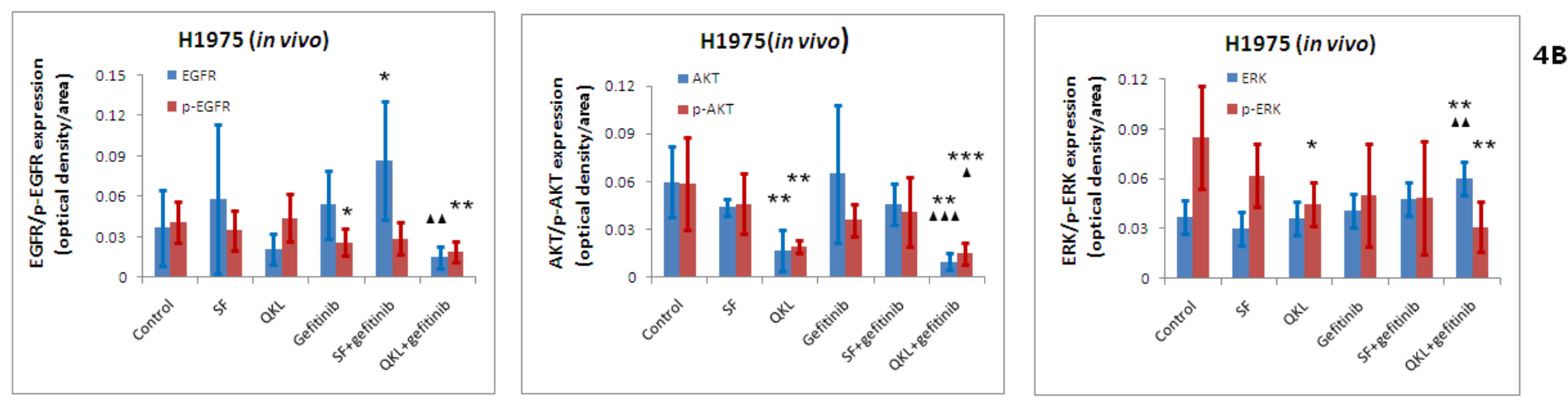

Figure 4

Western blot analysis didn't show clear mechanism of combination effect between gefitinib and Shenfu /Qingkailing injection in A549 cell line in vitro (4A). Data are presented as the mean \pm standard deviation of three independent experiments. Immunohistochemistry analysis indicated that EGFR phosphorylation inhibition and the downstream PI3K/AKT and RAS/RAF/ERK pathway inhibition served an important role in the synergism effect between QKL oral solution and gefitinib in $\mathrm{H} 1975$ xenograft transplanted nude mice (4B). Data are presented as the mean \pm standard deviation. ${ }^{*} P<0.05, * * P<0.01$ and ${ }^{*} * * P<0.001$ vs. control. $\mathbf{\Delta} P<0.05, \boldsymbol{\Delta} \mathbf{\Delta} P<0.01$ and $\mathbf{\Delta} \mathbf{\Delta} \mathbf{\Delta} P<0.001$ vs. gefitinib alone. SF, Shenfu injection (in vitro) or decoction (in vivo); QKL, Qingkailing injection (in vitro) or oral solution (in vivo). 